\title{
Creating a Business Process Monitoring System “A-IOMS” for Software Development
}

\author{
Hirotake Sakai, Kakuro Amasaka \\ Aoyama Gakuin University, Sagamihara, Japan
}

\begin{abstract}
In this paper, the authors establish the Amalab-integrated operation management system (A-IOMS) as a new way to carry out software development and monitor business processes. This new system puts the previously constructed the Amalab-project planning navigation system (A-PPNS) to work throughout the entire organization. The A-IOMS has several key elements. Specifically, the authors have constructed: (1) a Hi-reliability estimation system diagnostic tool that pinpoints problems with software development estimates and offers a prescription for resolving them; (2) a QA table map that allows for an immediate, thorough, and consistent quality assurance process; (3) a system for sharing software development schedules throughout the organization; and (4) a technological asset completion reporting system for passing down and cultivating software development technologies and strategically expanding them. The authors verified the effectiveness of the A-IOMS at company A, a systems integration company, and the given outcomes were obtained.
\end{abstract}

Keywords: software development, business process, A-IOMS, project management

\section{Introduction}

The tasks associated with software development typically rely on the experiential knowledge (unspoken work information) of the developers. As a result, most companies have not sufficiently shared this work information throughout their organizations (Otsuka, 2008).

In response, the authors (Sakai, Waji, Nakamura \& Amasaka, 2011) have identified the necessity of making software developers' knowledge explicit and constructed the A-PPNS (Amalab-Project Planning Navigation System) as a means of systematically reorganizing software development knowledge and work processes - and the given outcomes were obtained. In this paper, the authors establish the A-IOMS (Amalab-Integrated Operation Management System) as a new way to carry out software development and monitor business processes.

This new system puts the previously constructed A-PPNS to work throughout the entire organization. The A-IOMS has several key elements. The first four are core systems: (1) a subsystem to support optimization of work estimates; (2) a schedule monitoring system; (3) a diagnostic system for optimizing quality, cost, and delivery (QCD); and (4) a system for strategically accumulating and using technology. The fifth element is an application system for operation management, which is designed to link these four core systems in an

Hirotake Sakai, Graduate Student, School of Science and Engineering, Aoyama Gakuin University.

Kakuro Amasaka, Professor and Ph.D., School of Science and Engineering, Aoyama Gakuin University.

Correspondence concerning this article should be addressed to Kakuro Amasaka, 5-10-1, Fuchinobe, Chuo-ku, Sagamihara-shi, Kanagawa-ken, 252-5258 Japan. E-mail: sakai.hirotake@gmail.com. 
integrated way and strategically expand them.

Specifically, the authors have constructed: (1) a Hi-Reliability Estimation System diagnostic tool that pinpoints problems with software development estimates and offers a prescription for resolving them; (2) a QA table map that allows for an immediate, thorough, and consistent quality assurance process; (3) a system for sharing software development schedules throughout the organization; and (4) a technological asset completion reporting system for passing down and cultivating software development technologies and strategically expanding them.

The authors verified the effectiveness of the A-IOMS at Company A, a systems integration company, and the given outcomes were obtained.

\section{Software Development Work: Current State and Issues}

While the progress of information technology has been remarkable, it often relies upon the implicit work of those in charge of software development (software development engineers) and faces issues such as the sharing of implicit knowledge and continuous process management (Otsuka, 2008; Galorath \& Evans, 2006; Suzuki, 2002; Software Engineering Center of Information-Technology Promotion Agency, 2006).

The authors' survey of software development project activities in the companies has revealed the following points (Sakai et al., 2011; Nakamura, Enta \& Amasaka, 2011):

(1) Estimating: As a characteristic example, project estimating is conducted with a reliance upon the implicit rules of thumb (implicit knowledge) built up over the years by experienced and skilled engineers. In cases where young engineers perform the estimating, they rely on discussions with the skilled engineers;

(2) Quality assurance: In many cases, one or more quality assurance engineers are involved with a project to conduct quality reviews. However, the methods of review rely upon the rules of thumb (implicit knowledge) of those quality assurance engineers;

(3) Progress tracking and reporting: The business tools essential for tracking and reporting project progress have become increasingly diverse and sophisticated in recent years. However, simple progress tracking via generic Excel files is common. The quality of reporting is insufficient, and as a result, cases are seen in which the quality of reports made to project managers at progress meetings is insufficient;

(4) Completion reports: Cases are seen in which, with staff pressured by project work, the level of standardization in completion reports is not high. As a result, the items that should be recorded in post-development completion reports are left unsettled in many cases. Completion reports tend toward the implicit, easily influenced by the competence and discretion of practitioners and relying on antiquated rules of thumb. As a result, the level of intellectual property is insufficient, and is not fully exploited for the transmission and development of technology.

\section{Creating A-IOMS to Reform Software Development Work}

Mittas and Angelis (2010) proposed a formal framework covering different aspects of the estimation process such as the calibration of the prediction methodology, the identification of factors that affect the error, the investigation of errors on certain ranges of the actual cost and the examination of the distribution of the cost for certain errors. Ross, Weill, and Robertson (2006) claimed that core business processes existed within companies, and that these business processes must be continuously improved.

In a similar manner, the authors and Kato (Amasaka, 2005; Kato, 2006; Kojima \& Amasaka, 2011) have 
demonstrated the construction and validity of an intelligent information system that contributes to the reform of various business processes in both manufacturing and non-manufacturing industries. In a similar manner, the authors (Mihara, Nakamura, Yamaji, \& Amasaka, 2010) discussed the improvement of business processes and the validity of intelligent information systems for the reform of software development work.

Based on these findings, the authors create a new methodology to reform previously implicit software development work: the Business Process Monitoring System, A-IOMS (See Figure 1).

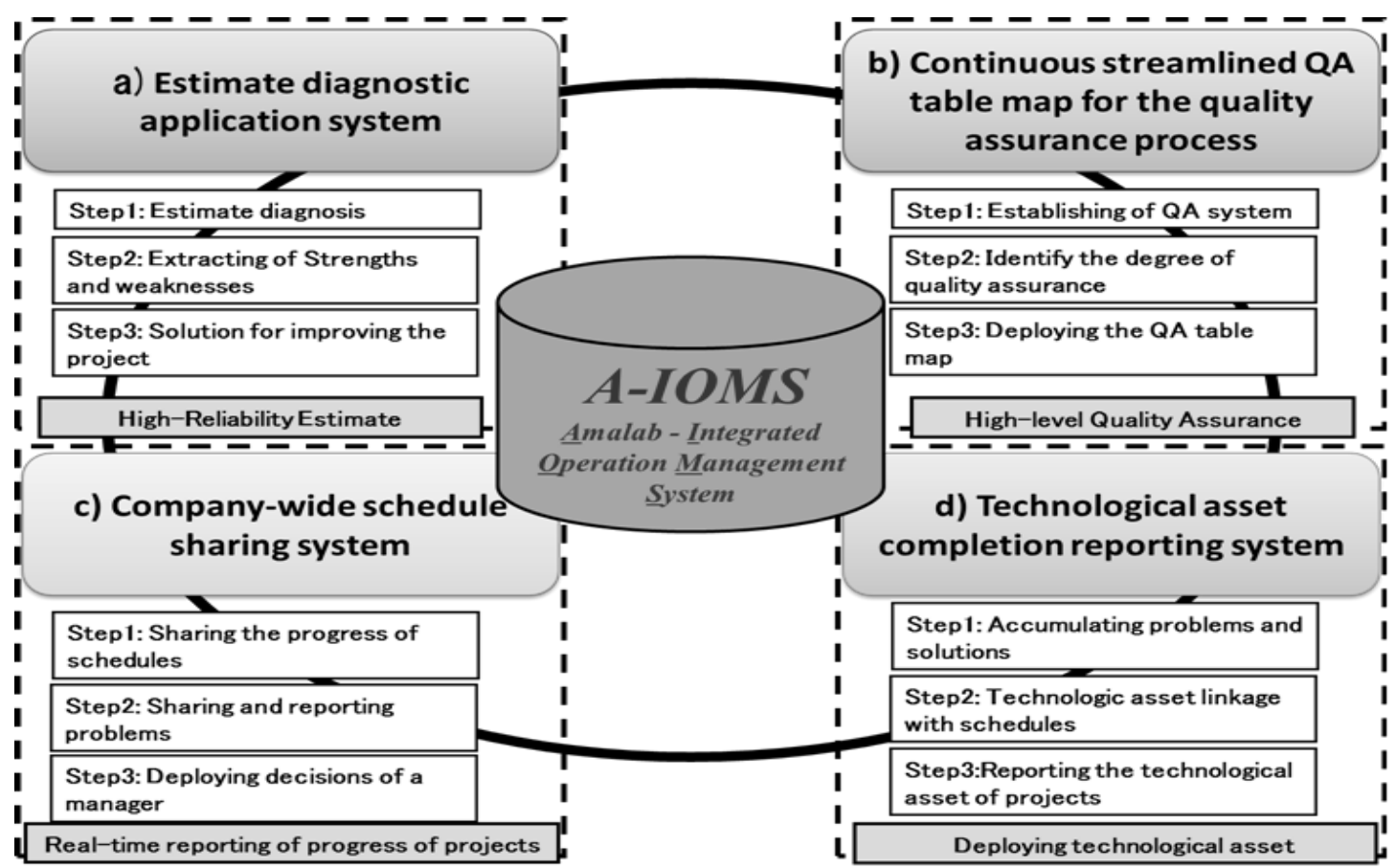

Figure 1. The business process monitoring system "A-IOMS”.

A-IOMS comprises four core systems to overcome the four challenges of software development work, as described in the previous section. The guidelines for solving each of the issues are outlined below:

(1) Estimating: To achieve precise estimating that avoids conflict with customers, we establish an estimate diagnostic model using statistical analysis, and construct an estimate diagnostic application system;

(2) Quality assurance: A quality assurance system was established for the entire process, construct a continuous streamlined a QA table map showing the degree of assurance of the process, and make explicit quality assurance engineers' implicit knowledge and points for review;

(3) Progress tracking and reporting: From managers to developers, an improvement was achieved in the quality of progress tracking and reporting through the use of integrated business tools;

(4) Completion reports: The authors achieve standardization of completion reports into a unified format, and at the same time, simplify the completion report at the time of project closure using in-development progress reports, and thus achieve the transmission and development of technology.

\section{Estimate Diagnostic Application System}

In this system the authors focused on estimating, a crucial item even in initial planning. They defined "high-reliability estimating" as precise estimating that avoids customer conflict, and constructed a high-reliability estimate diagnostic model using multivariate statistical analysis. 
Specifically, as shown in Figure 2, a 16-item questionnaire was analyzed aimed at engineers engaged in software development with a multiple regression, and constructed a diagnostic model to support the creation of high-reliability estimates. Through this system, the impact of development team performance on productivity is made clear, and by enhancing the performance of the whole team toward the goal of productivity, it is possible to achieve high-reliability estimates.

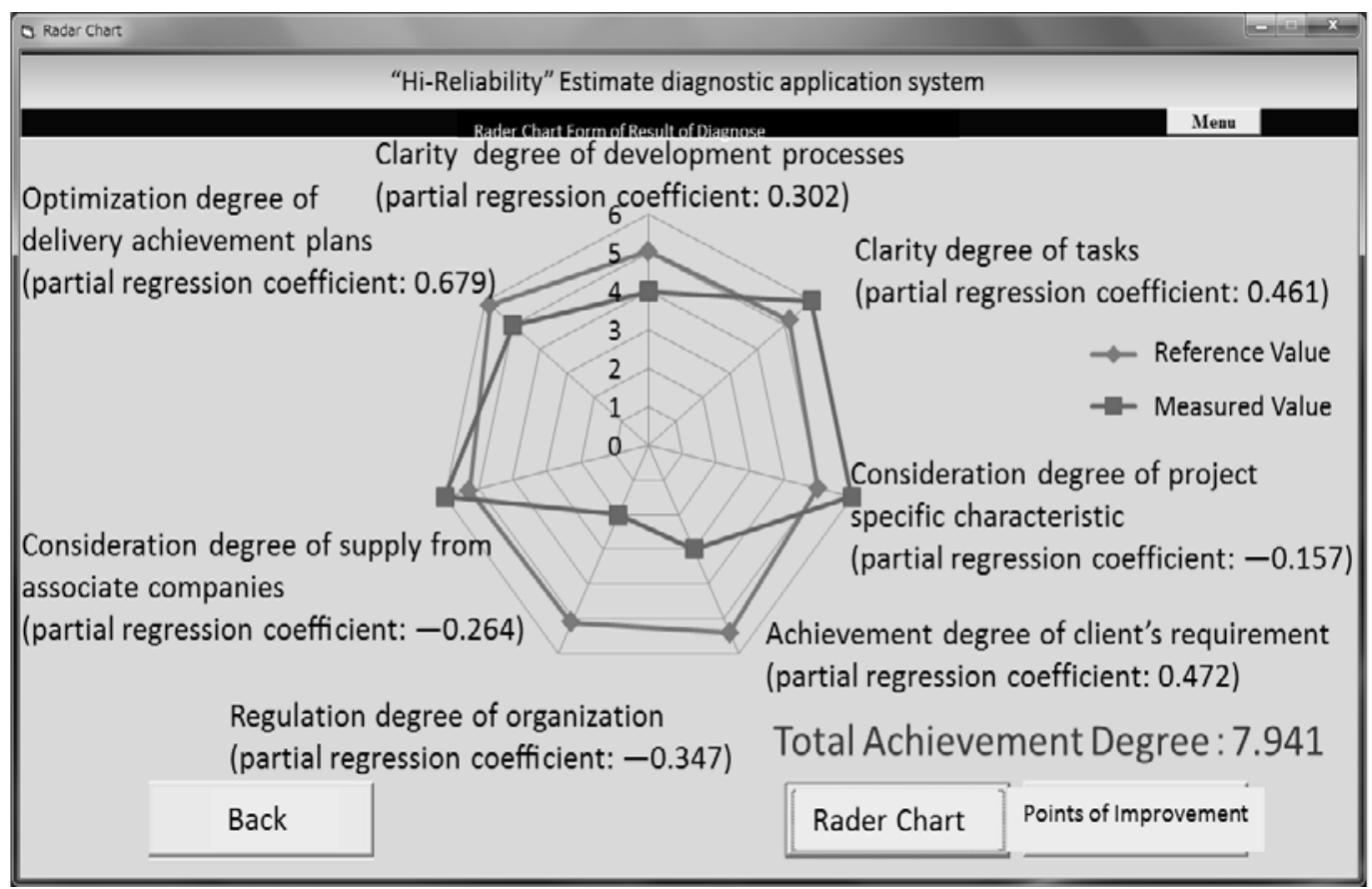

Figure 2. The estimate diagnostic application system.

\section{Continuous Streamlined QA Table Map for the Quality Assurance Process}

In this system, the authors established a quality assurance system for the entire process, and constructed a continuous streamlined QA table map showing the degree of assurance of the process. The QA table map shown in Figure 3 is a continuous streamlined quality assurance technique supported by Failure Mode and Effect Analysis (FMFA) and matrix diagrams.

This table map quantitatively assesses the importance of processes, with the entire process on the vertical axis and quality characteristics on the horizontal axis, and degree of assurance marked at the intersection of the vertical and horizontal axes. By sharing and leveraging this table map, not only quality assurance engineers but also the entire team, including development engineers, can carry out continuous streamlined quality assurance and improve the quality of the entire process.

\section{Company-Wide Schedule Sharing System}

In this system, a database was constructed for sharing schedules and progress status inside and outside of the development team. Specifically, as shown in Figure 4, this adds reporting functions for basic information such as detailed work items, rate of progress, and number of days delayed, and for generated issues. With this database, the system allows progress reports used in progress meetings to be output in a unified format, thus enabling smooth progress reporting. In addition, various managerial positions can view project progress and issues in real time, and can promptly issue instructions for improvement to the development engineers. 


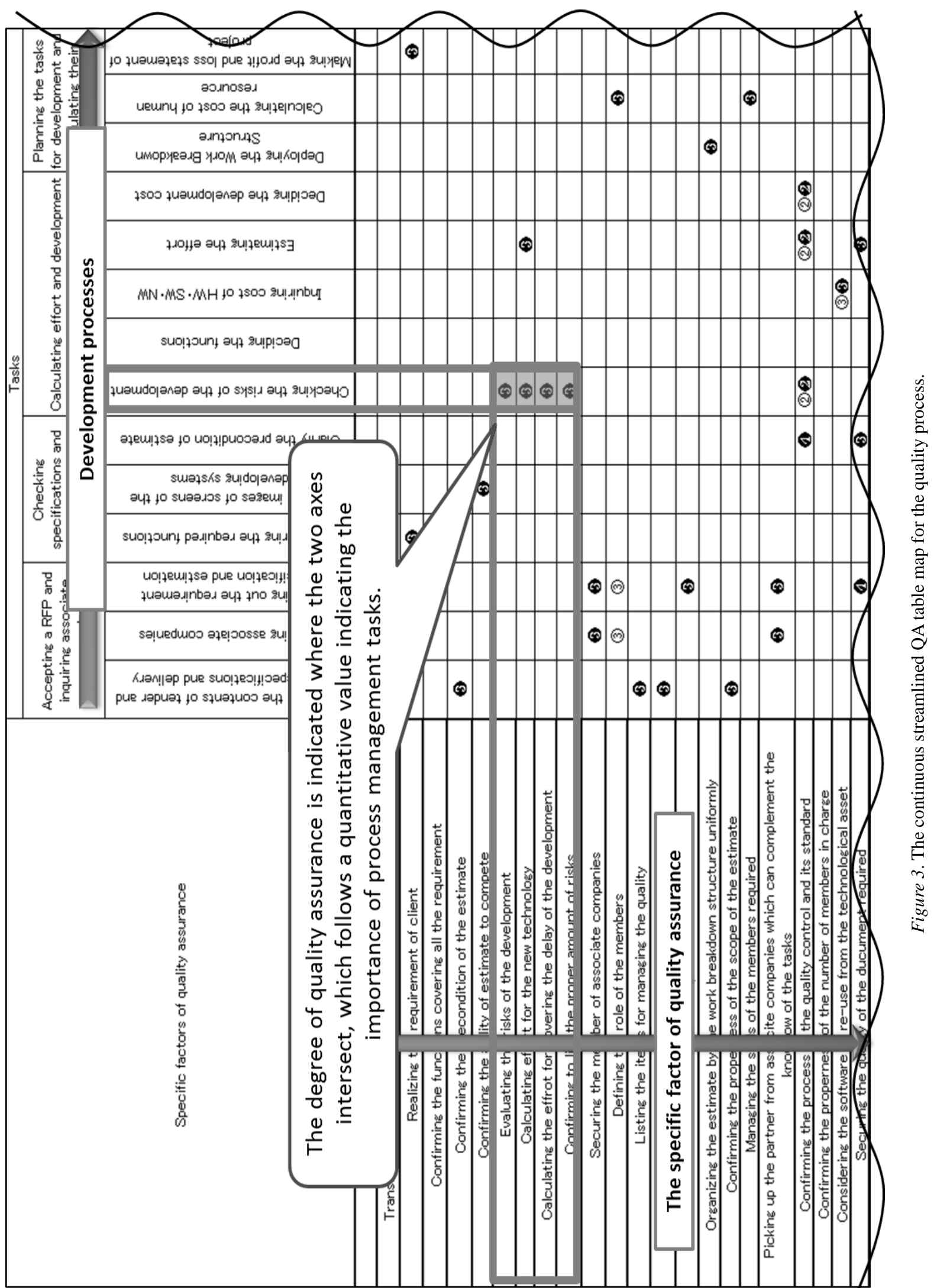




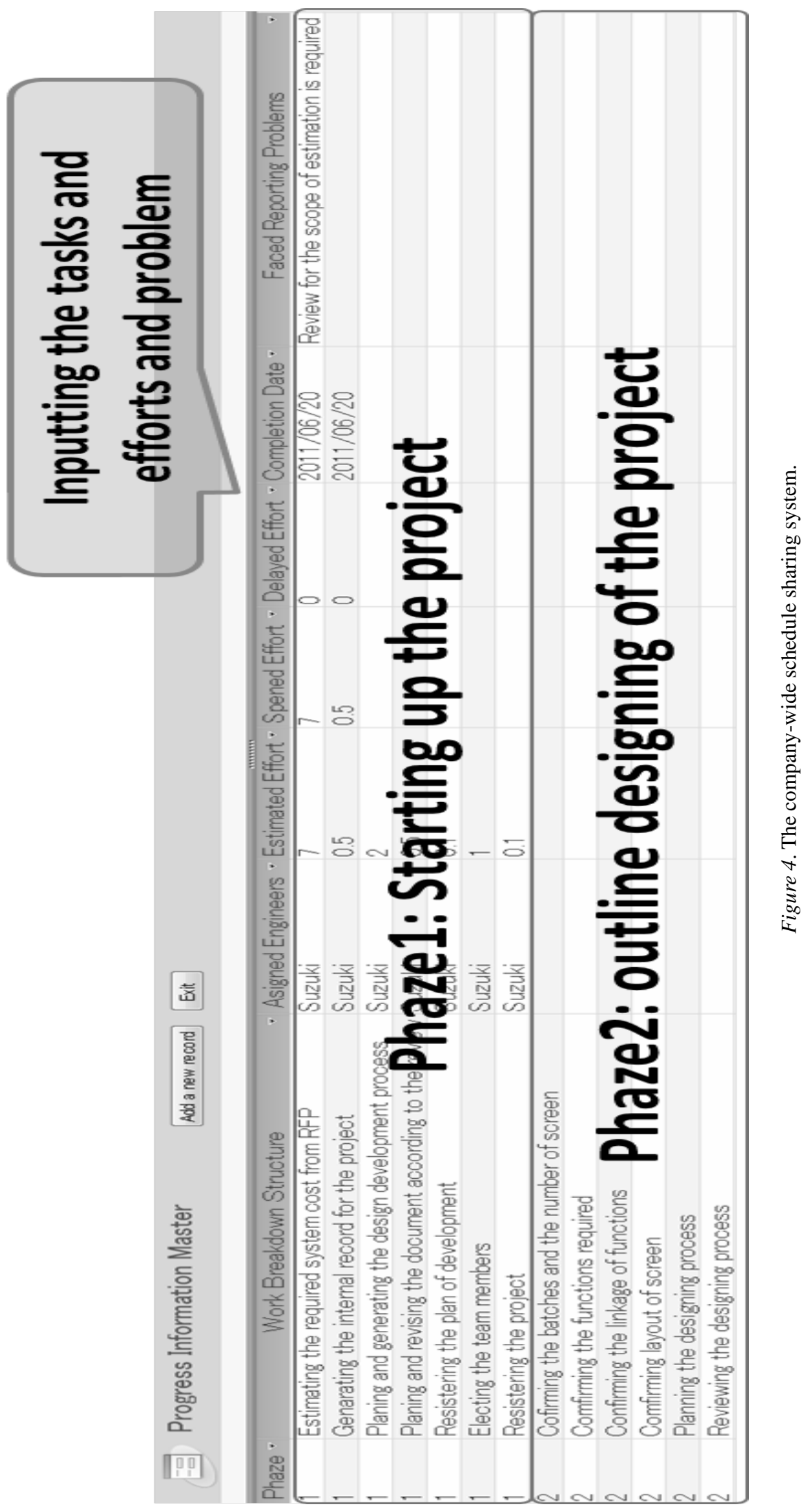




\section{Technological Asset Completion Reporting System}

This system takes the issues accumulated in the company-wide schedule sharing system together with in-progress project schedules, and links these with the completion reports logged after the close of the project, thus assetizing technology. By viewing the reports shown in Figure 5, it is possible to gain past technical information and business knowledge, and to employ these in development and expansion of current projects.

In addition, items that should be incorporated into completion reports have in the past differed by engineer. By utilizing the in-development schedule database and formatting of completion reports, what was previously implicit can be accumulated internally in reports of uniform quality, and the technology of the completed project can be assetized.

\section{Validity of the Business Process Monitoring System A-IOMS}

The authors verified the validity of the established A-IMOS through the system integrator company A, and the given outcomes were obtained. Specifically, the constructed A-IOMS was employed in a project by company A, and feedback was collected from multiple engineers and managers. Furthermore, in order to confirm contributions to productivity improvements, the difference was analyzed between the predicted and actual values of the applicable project's scale, and demonstrated validity.

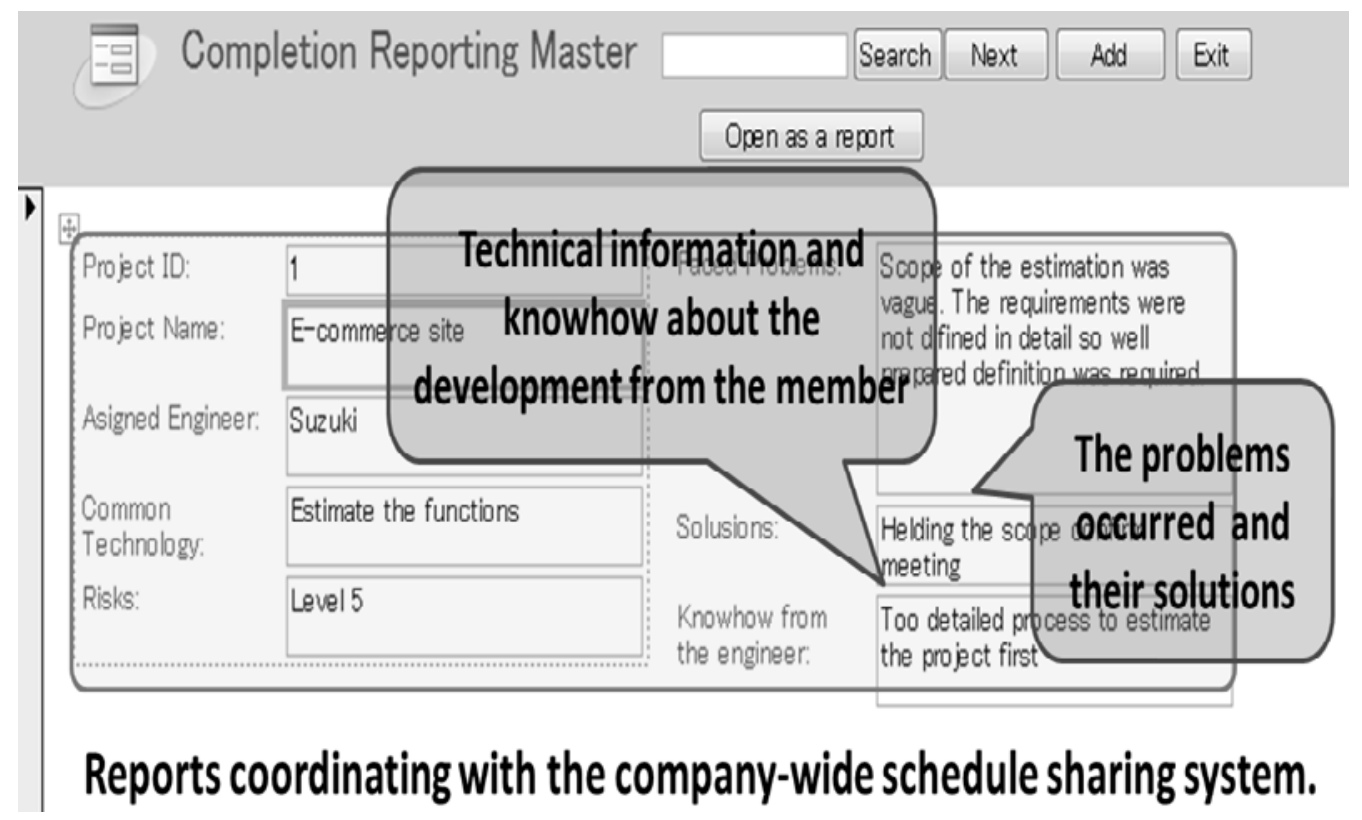

Figure 5. The technological asset completion reporting system.

\section{Conclusion}

In this paper, the Business Process Monitoring System A-IOMS was established to reform business processes by capturing implicit knowledge of software development in the four processes of estimating, quality assurance, development schedule and progress reporting, and post-development completion reports. The authors verified the validity of A-IOMS through software development in company A, and obtained the given outcomes. In this paper, we established a methodology for business process reform and a system for its achievement through A-IOMS. Prospects for further research include improving the quality of accumulated information, and more sophisticated methods of exploiting that information. 


\section{References}

Amasaka, K. (2005). Constructing a customer science application system “CS-CIANS”-Development of a global strategic vehicle "Lexus” utilizing New JIT. WSEAS Transactions on Business and Economics, 2, 135-142.

Galorath, D., \& Evans, W. (2006). Software sizing, estimation, and risk management. Auerbach Publications.

Kato, K. (2006). Visualization of work processes on a research industry (in Japanese) (Graduation thesis, Aoyama Gakuin University).

Kojima, T., \& Amasaka, K. (2011). The total quality assurance networking model for preventing defects: Building an effective quality assurance system using a total QA network. International Journal of Management and Information Systems, 15(3), $1-10$.

Mihara, R., Nakamura, M., Yamaji, M., \& Amasaka, K. (2010). Study on business process navigation system “A-BPNS”, International Journal of Management and Information Systems, 14(2), 51-58.

Mittas, N., \& Angelis, L. (2010). Visual comparison of software cost estimation models by regression error characteristic analysis, Journal of Systems and Software, 83(4), 621-637.

Nakamura, M., Enta, Y., \& Amasaka, K. (2011), Constructing a model to assess the success of information sharing between customers and vendors in software development. Proceedings of International Conference on Business Management 2011, Miyazaki Sangyo-Keiei University, Japan (pp. 109-118).

Otsuka, H. (2008). System development methodology making effective use of intellectual property. Unisys Technology Review (in Japanese), 28(2), 3-14.

Ross, J., Weill, P., \& Robertson, D. (2006). Enterprise architecture as strategy: Creating a foundation for business execution. Harvard Business Press.

Sakai, H., Waji, Y., Nakamura, M., \& Amasaka, K. (2011). Establishment of “A-PPNS”: A navigation system for regenerating the software development business. Industrial Engineering and Management Systems, 10(1), 44-54.

Software Engineering Center of Information-Technology Promotion Agency (2006). Guidebook of the Software Development Estimation (in Japanese), 3-97.

Suzuki, M. (2002). Information science and engineering (in Japanese). Saience-Sha Co., Ltd. Publishers. 